NASA Technical Memorandum 102282

\title{
Compensation for Effects of Ambient Temperature on Rare-Earth Doped Fiber Optic Thermometer
}

G. Adamovsky, J.L. Sotomayor, and M.J. Krasowski

Lewis Research Center

Cleveland, Ohio

and

J.G. Eustace

John Carroll University

Cleveland, Ohio

Prepared for

Fiber Optic and Laser Sensors VII

sponsored by the Society of Photo-Optical Instrumentation Engineers

Boston, Massachusetts, September 5-8, 1989

\section{NRSA}

(HA SA-TY-102282) COAPEUS ATION POR EFFECTS 


\author{
Compensation for effects of ambient temperature on \\ rare-earth doped fiber optic thermometer \\ G. Adamovsky, J.L. Sotomayor, and M.J. Krasowski \\ NASA Lewis Research Center \\ Cleveland, Ohio 44135
}

and

J.G. Eustace

John Carroll University

Physics Department

Cleveland, Ohio 44118

\begin{abstract}
Variations in ambient temperature have a negative effect on the performance of any fiber optic sensing system. A change in ambient temperature may alter the design parameters of fiber optic cables, connectors, sources, detectors, and other fiber optic components and eventually the performance of the entire system. The thermal stability of components is especially important in a system which employs intensity modulated sensors. Several referencing schemes have been developed to account for the variable losses that occur within the system. However, none of these conventional compensating techniques can be used to stabilize the thermal drift of the light source in a system based on the spectral properties of the sensor material. The compensation for changes in ambient temperature becomes especially important in fiber optic thermometers doped with rare earths. Thus, the purpose of this work is to search and analyze different approaches to solving this problem.
\end{abstract}

\title{
1. INTRODUCTION
}

Spectral sensors are those that respond to the measured parameter by modulating the spectrum of an optical signal according to spectral characteristics of the sensor material. The spectral sensors are governed by different physical phenomena, among which absorption is the most commonly used. The measured parameter (temperature, pressure, etc.) alters the absorption spectrum of the sensor material and changes the transmission of the system accordingly. Varlous spectral sensors have already been discussed in numerous publications. $1-4$

The inherently selective optical properties of the spectral sensors make the entire system heavily dependent on the stability of the light sources used. Light emitting diodes (LED) have relatively narrow spectral bandwidths. A change in the ambient temperature causes the LED to change its central wavelength, spectral bandwidth, and emitted power. This results in changes in the return signal that are not a result of changes in the measurand. The signal processing electronics do not discriminate between transmission variations due to changes in the measured parameter and those due to changes in the ambient conditions. Thus the ambiguity results in an increased error and limits applicability of spectral sensors. 
Ambient temperature has the greatest effect on the spectral and power characteristics of the light sources. The spectral stability of the sources becomes especially important in airborne fiber optic sensing systems. Optical sources for aircraft applications are expected to operate over a temperature range from -55 to $125^{\circ} \mathrm{C}$. In this work we will discuss different ways to compensate for or at least minimize the effects of a changing ambient temperature on the performance of a fiber sptic thermometer based on absorption in rare-earth doped materials.

Due to a specific electron configuration of the rare earths, all elements of this group exhibit a strong temperature dependent absorption after being incorporated into a host material as impurity ions. The absorption spectrum of the rare earth ion varies from element to element and also depends on the host medium. 5,6 Neodymium is one of the most attractive materials to use in temperature sensing systems, mainly because its absorption spectrum lies in the same spectral region as commercially available high power GaAlAs LEDs. At the same time accurate temperature measurement can be achieved over a broad range of temperatures.

\section{EFFECT OF TEMPERATURE ON LED PERFORMANCE}

The spectral distribution of energy radiated from an LED during an electronhole recombination process is governed by the Boltzmann relation and is therefore temperature dependent. The theory of this phenomenon is explained in detail in numerous publications on semiconductors, solid state physics, and optical communication.7-10 It has also been shown that the observed emission spectra of LEDs are much more symmetrical around the central wavelength than one would expect from theoretical predictions. For reasons of simplicity, it is conventionally accepted to treat the emission spectrum of an LED as a Gaussian distribution. The central wavelength of the emission spectrum is determined largely by a direct band gap in a binary semiconductor material. GaAs, InSb, and InP are examples of III-V binary compounds widely used for manufacturing LEDs. Tuning of the central wavelength can be obtained by varying the composition of components in more complex conflgurations like ternary (GaAlAs) or quaternary (InGaAsP, InGaAsSb) systems. In all cases, the temperature has a direct effect on the band gap and its increase causes the central wavelength to shift toward longer wavelengths. The spectral half-width of the radiation is also affected by the temperature. Theoretical and experimental studies done on optical characteristics of semiconductor light sources have shown that their temperature dependent properties obey some exponential relationship. A prolonged operation of LEDs at elevated temperatures also causes degradation in their performance. The degradation manifests itself in a quasi-exponential decrease in output power with time. The change in the spectral characteristics of an LED operated at $100 \mathrm{~mA}$ continuous current and ambient temperature $\mathrm{Ta}=90^{\circ} \mathrm{C}$ is demonstrated in $\mathrm{Fig}$. 1 . The figure shows spectra measured immediately after the ambient temperature reached $90{ }^{\circ} \mathrm{C}$, after 72 hours, and after 168 hours of operation at this temperature.

Figure 2 shows LED spectra measured at different ambient temperatures. To characterize the thermal sensitivity of LEDs such incremental parameters as a thermally induced changes in the central wavelength $\Delta \lambda_{c} / \Delta T$, spectral bandwidth $\Delta(\Delta \lambda) / \Delta T$, and emitting power $\Delta P / \Delta T$ are often used. These parameters are sometimes given by LED manufacturers in product specifications, and their values depend on both the material and the fabrication process used. 


\section{COMPENSATION FOR CHANGES IN EMITTED POWER}

Among the parameters mentioned above, the temperature effects on LED emitted power is the easiest one for which to compensate. Variations in the emitted power propagate through the system and affect its performance the same way variable losses do. Different techniques for compensation have been proposed.11 The most commonly used compensating technique is based on a two wavelength referencing scheme. 12,13 In this compensating technique, one wavelength lies in the range of spectral sensitivity of the sensor. The other wavelength is outside this range. Thus, the ratio of signals coming back from the sensor is independent of variable losses through the system. If the parameter $\Delta P / \Delta T$ has the same value at both wavelengths, this ratio would also be independent of the variations in the emitted power. However, in a system with two LEDs the parameter $\Delta P / \Delta T$ is usually different for each source. In this case additional referencing may be used.

An example of a sensing system with dual wavelength referencing and power level compensation is shown in Fig. 3. Two LEDs with different wavelengths, $\lambda_{1}$ and $\lambda_{2}$, are used to generate a signal and a reference channel. The LEDs are driven by a pulse generator in such a way that pulses of radiation emitted by the LEDs are multiplexed in time. This permits using the same photodetector for both the signal and the reference channels. An initial signal that consists of two pulses enters the system through a 2 by 2 coupler. The coupler splits the signal through two ports. One port directs the pulses towards the sensor and the return signal is detected by a photodetector (PDS). The signal that reaches this photodetector consists of a periodic double pulse, with one pulse at the wavelength $\lambda_{1}$ having an amplitude Vsi and the other one at the wavelength $\lambda_{2}$ having an amplitude $V_{s_{2}}$. The other port is used to direct a portion of the initial signal towards another photodetector. PDr, for the power level compensation. This photodetector sees pulses of amplitudes $V r$ l and $V r_{2}$ at wavelengths $\lambda_{1}$ and $\lambda_{2}$, respectively. The resultant signal used to retrieve information about the measured parameter can be written in the form:

$$
V=V s_{1} V r_{2} / V s_{2} V r_{1}
$$

where $V_{s_{1}}$ and $V_{s_{2}}$ are amplitudes of signals coming from the sensor at wavelengths $\lambda_{1}$ and $\lambda_{2}$ respectively, and $V r_{1}$ and $V r_{2}$ are amplitudes of power level compensating signals at wavelengths $\lambda_{1}$ and $\lambda_{2}$ respectively, and is independent of variations in LED power levels. Experimental testing of this scheme has shown that an arbitrary decrease in the output power of any of the LEDs used to 70 percent of the initial power results in less than a 2 percent error in the final result.

An alternate approach involves time domain referencing.14-16 The principle of this technique is based on separation of the signal and reference channels in the time domain over the same fiber optic link. This is achieved by pulsing the source and comparing the amplitude of a reference pulse with the one returned from the sensor. The ratio of the pulse amplitudes is independent of the initial power level. Another compensating scheme that can be used involves contrast modulation of an interference pattern by an intensity sensor. 17

\section{COMPENSATION FOR CHANGES IN SPECTRAL DISTRIBUTION}

Because of the specific nature of spectral sensors, power level referencing alone is not sufficient for constructing a thermally stable sensing system. 
Therefore, in addition to power referencing, it is necessary also to stabilize the system against changes in the optical spectrum of the light source used. Fiber optic sensing systems stabilized against changes in spectral characteristics of LEDs due to changes in the ambient temperature can be grouped into three categories: systems with active compensation, systems with passive compensation, and systems without compensation.

\section{Systems with active compensation}

In systems with active compensation the spectral characteristics of the source are maintained by controlling the temperature of the junction directly. Two parameters are mainly involved in a thermodynamic process of heating an LED, an average current that passes through the junction and the ambient temperature of the device. Both the current and the ambient temperature contribute jointly to the thermal stahility of the light sources. LED spectra at room temperature and different dc currents are shown in Fig. 4. It is obvious that in order to stabilize the spectrum at room temperature the average current should be small. This can be achieved by pulsing the source instead of employing continuous $d c$. Lower pulse duty cycles yield lower average currents and lessen the thermodynamic disturbance (heat). 18 This results in reduced spectral changes in the LED radiation. Thus, the simplest form of active compensation could involve changing the average current through the junction in accordance with the change in the ambient temperature. However, this approach has restrictions because of limited dynamic range of the current-wavelength dependency.

Another active compensation technique involves heating or cooling the device to temperatures which are basically outside the reach of ambient temperature excursions or holding it at a desired set point temperature anywhere within the operating range of the device.

Heating of the LED is achieved with either resistive or thermal electric devices. Resistive heaters fall into two general categories: resistive strip and positive temperature coefficient (PTC) thermistors. In the resistive strip the current passes through a resistive element which dissipates heat generated in the process. The LED assembly is thermally connected with the strip and is in turn heated. PTC thermistors are passive devices whose electrical resistance rises with temperature. The device dissipates the power as heat. The heat in turn raises the resistance of the thermistor. As a result the dissipated power decreases. This internal feedback allows stable operation without external control.

Another form of active compensation involves thermal electric devices (TED). The principle of these devices is based on the dissimilar thermodynamic properties of two materials configured in such a way that they form two junctions. A voltage applied to these junctions creates a temperature difference between the two junctions. This phenomenon is known as the Peltier effect. The direction of heat flow through the device is dependent on the polarity of the input electrical current. Therefore heating can be achieved by applying current in one direction, cooling by applying it in the other. In the heating mode a large portion of the heat produced by the device is due to its resistive component. The remainder must be pumped from the environment and this requires a great deal of electric power; more so than the schemes mentioned above. Also, the device loses efficiency as the temperature of the outside environment drops. 
The efficlency of the TED as a cooling element is even lower because the resistive component in the device is producing heat regardless of the direction of the current flow. Therefore in the cooling mode the power applied to the TED must overcome its self heating and also cool the attached LED. However for its size and given the fact that it has no moving parts or restrictions on physical orientation, the TED is an ideal cooling device for electronic components.

\subsection{Systems with passive compensation}

In systems with passive compensation the average current through the junction remains the same and the compensation is achleved in the signal processing hardware. The signal processing hardware provides compensation based on knowledge of values of LEDs spectral characteristics, such as the central wavelength and bandwidth, at different temperatures. The information about the spectral parameters can be obtained either indirectly by measuring the ambient temperature and predicting these parameters or directiy by measuring the position of the central wavelength and the bandwidth.

A schematic of an indirect passive compensation scheme is presented in Fig. 5 . A look up table (LUT) which contains the values of the spectral parameters for the specific LED used versus temperatures is positioned in the read only memory (ROM). The signal that comes back from the sensor is detected by the photodetector (PD), analyzed in the preprocessor (PPC), and sent to the microprocessor ( $\mu P$ ). Values of the spectral parameters of the LED at the measured ambient temperature Ta are read from the LUT. A major problem lies in the fact that a replacement of one LED by another one could lead to a reprogramming of the ROM and to a recalibration of the entire sensing system. Despite the fact that variations in the source intensity can be compensated by the power referencing technique as mentioned above, any other changes in the source spectral characteristics due to aging and degradation are not eliminated by this technique.

A direct passive compensation technique involves the continuous measurement of the spectral parameters of the LEDs used and processing these data with a microprocessor. A schematic diagram of a sensing system using direct passive compensation is shown in Fig. 6. A portion of the radiation emitted by the light source is spread in the wavelength domain by an appropriate dispersing element (DE). A photodetector array (PDA) sees this dispersion as a spectral intensity distribution along the array. Thus the shape of the distribution and its position can be easily detected. A signal conditioner (SC) takes the information from the array and converts it into data acceptable to a microprocessor ( $\mu P)$. The data from the sensor is also sent to the microprocessor via a photodetector (PD) and a preprocessor (PPC). This technique permits an accurate tracking of the entire spectral intensity distribution. Thus, additional power level referencing can be avoided. Obviously, this technique provides a better compensation than the previous one. However a sensing system with direct passive compensation would be more complex, and therefore, possibly less reliable.

\subsection{Systems without compensation}

Fiber optic sensing systems without compensation are designed to tolerate all variations in the spectral distribution of the LED radiation over a specified range of ambient temperatures. 
The best known configuration of a Nd-based temperature sensing system without compensation involves restricting the optical spectrum of the source using optical filters.19 This technique provides effective stabilization and has been used successfully on several occasions. However the employment of filters also has drainbacks. Transmission of the filters is low and usually does not exceed 60 percent. This places demands on the power budget and establishes a requirement either to use more powerful LEDs or to increase the operating current. In addition to this, the spectral window of the filter itself is affected by the ambient temperature. This thermal sensitivity is greater for filters with narrower spectral windows. Among the other disadvantages of using optical filters are possible alignment problems and reduced reliability due to use of additional components.

Another approach to building a Nd-doped thermometer without compensation involves using parts and components that are insensitive to changes in the ambient temperature. The sensor itself and the LED used are the primary targets for engineering efforts to build a system insensitive to ambient temperatures.

The sensor could be designed to tolerate some variations in the LED emission spectrum. The absorption. spectrum of $\mathrm{Nd}$-doped material depends on both the concentration of $\mathrm{Nd}^{3+}$ ions and the host used. An increase in the concentration leads to overlapping of individual absorption lines and the formation of solid absorption bands. Figure 7 presents measured transmission spectra of $6 \mathrm{~mm}$ thick samples of glass doped with $3.5,6.0$, and $8.0 \mathrm{wt} \% \mathrm{Nd}_{2} \mathrm{O}_{3}$. Broader absorption bands and sharper boundaries between the bands are clearly associated with a larger concentration. However the described approach permits only partial stabilization over a small range of ambient temperatures because the middle points of the neighboring absorption and transmission bands in the spectral region of interest ( 700 to $900 \mathrm{~nm}$ ) are separated by approximately $30 \mathrm{~nm}$. Thus, for an LED with $\Delta \lambda_{C} / \Delta T=0.1 \mathrm{~nm} /{ }^{\circ} \mathrm{C}$, $\Delta \lambda=20 \mathrm{~nm}$, and $\Delta(\Delta \lambda) / \Delta T=0$, compensation would be possible for an approximate range of ambient temperatures $\mathrm{Ta}=\mathrm{To}=5{ }^{\circ} \mathrm{C}$. This prediction has been made under the assumptioris that boundaries between the bands have a rectangular shape and the LED central wavelength at room temperature To lies in the middle of the absorption band.

Host materials also assist in the broadening of the absorption bands. Hosts with crystalline structures of lower symmetry would exhibit broadening and overlapping of the absorption bands. On the other hand, fine structures of absorption asscciated transitions could be observed in host materials with highly symmetrical structures. Recent reports on such materials with low symmetry hosts as Nd:YVO and Nd:BeL have shown significant broadening of the absorption bands in 1 percent Nd-doped crystals.20-24 Because amorphous materials have the lowest symmetry. glass would be one of the best candidates as a host material.

As regards the source it is obvious that the sensitivity of the system to ambient temperature can be reduced if the spectral bandwidth is significantiy greater than the spectral region of interest where the absorption bands of $\mathrm{Nd}^{3+}$ ion lie. To show the effect of the spectral bandwidth, two LEDs with the same central wavelength of $810 \mathrm{~nm}$ and different spectral bandwidths of 30 and $100 \mathrm{~nm}$ have been chosen for the study. Then assuming that the initial emitted power at room temperature for both LEDs is the same, $\Delta \lambda_{c} / \Delta T=0.2 \mathrm{~nm} /{ }^{\circ} \mathrm{C}$, and $\Delta(\Delta \lambda) / \Delta T=0.1 \mathrm{~nm} /{ }^{\circ} \mathrm{C}$, a total power $P$ that reaches a photodetector has been computed as a function of the sensor temperature $T s$ for ambient temperatures $\mathrm{Ta}=-40{ }^{\circ} \mathrm{C}, \mathrm{Ta}=25{ }^{\circ} \mathrm{C}$, and $\mathrm{Ta}=75^{\circ} \mathrm{C}$. The results of the computations are shown in Table I. It is seen from the table that the deviation in the computed power for the LED with $\Delta \lambda=100 \mathrm{~nm}$ 
at $\mathrm{Ta}=-40^{\circ} \mathrm{C}$ and $\mathrm{Ta}=75^{\circ} \mathrm{C}$ does not exceed 1 percent of the power at $\mathrm{Ta}=25^{\circ} \mathrm{C}$ over the range of $\mathrm{Ts}$ from 0 to $300^{\circ} \mathrm{C}$. On the other hand, for the LED with $\Delta \lambda=30 \mathrm{~nm}$ the corresponding changes in the computed power rapidiy become intolerable.

\section{CONCLUSION}

The preliminary results of the study have shown that despite a variety of compensating techniques, not all of them can be used in airborne applications due to either complexity, size, or power budget considerations. The most promising approach would involve an LED with a broad spectral bandwidth. A reference channel in this case could be constructed by either using another LED with a central wavelength in the spectral region outside the absorption bands of the $\mathrm{Nd}^{3+} \mathrm{ion}$, or employing one of the time domain or RF modulation referencing techniques. The current nonavailability of LEDs with sufficiently broad spectral bandwidths can be overcome by combining several LEDs with different central wavelengths and narrower bandwidths. This approach leads to a more complex system. However, the complexity can be reduced if, in addition, one of the forms of either active or passive compensation is used. This would permit the utilization of commercially available LEDs with narrower bandwidths. Experimental tests are planned in order to analyze fully the advantages and disadvantages of the compensating techniques discussed in this study.

\section{ACKNOWLEDGMENTS}

J.G. Eustace acknowledges the support of the NASA Lewis Research Center (NASA Grant NAG-3-984).

\section{REFERENCES}

1. K. Kyuma, S. Tai, T. Sawada, and M. Nunoshita, "Fiber-Optic Instrument for Temperature Measurement," IEEE J. Quantum Electron, vol. QE-18, pp. 676-679, Apr. 1982.

2. E. Theocharous, "Displacement and Temperature Multimeasurant Transducer," in Conference Proceedings OFS ' 84 - Second International Conference on Optical Fiber Sensors, R.T. Kersten and R. Kist, eds., Proc. SPIE 514, pp. 199-203, 1984 .

3. E. Snitzer, W.W. Morey, and W.H. Glenn, "Fiber Optic Rare Earth Temperature Sensors," in First International Conference on Optical Fiber Sensors, Conf. Publ. No. 221, Institution of Electrical Engineers, London, U.K., pp. 79-81, 1983.

4. M. Bacci, M. Brenci, G. Conforti, R. Falciai, A.G. Mignani, and A.M. Scheggi, "Thermochromic Transducer Optical Fiber Thermometer," Appl. Opt., vol. 25, pp. 1079-1082, Apr. 1, 1986.

5. G.H. Dieke and H.M. Crosswhite, "The Spectra of the Doubly and Triply Ionized Rare Earths," Appl. Opt., vol. 2, pp. 675-686, July 1963.

6. J.R. Thornton, W.D. Fountain, G.W. Flint, and T.G. Crow, "Properties of Neodymium Laser Materials," Appl. Opt., vol. 8, pp. 1087-1102, June 1969.

7. J.I. Pankove, Optical Processes in Semiconductors, Prentice-Hall, Englewood Cliffs, NJ, pp. 229-234, 1971. 
8. J. Gowar, Optical Communication Systems, Prentice-Hall, London, pp. 234-236, 1984 .

9. A. Chiari, M. Colocci, F. Fermi, Y. Li, R. Querzoli, A. Vinattieri, and W. Zhuang, "Temperature Dependance of the Photoluminescence in GaAs-GaAlAs Multiple Quantum Well Structures," Phys. Stat. Sol. B, vol. 147, pp. 421-429, 1988.

10. R.C. Goodfellow and R. Davis, "Optical Source Devices," in Optical Fibre Communications, M.J. Howes and D.V. Morgan, eds., John Wiley a Sons, pp. $27-106,1980$.

11. G. Adamovsky, "Referencing in Fiber Optic Sensing Systems," in Optical Techniques for Sensing and Measurement in Hostile Environments, C.H. Gillespie and R.A. Greenwell, eds., Proc. SPIE 787, pp. 17-23, 1987.

12. B.E. Jones and R.C. Spooncer, "Two-Wavelength Referencing of an Optical Fibre Intensity-Modulated Sensor," J. Phys. E: Sci. Instrum., vol. 16, pp.

1124-1126. Nov. 1983.

13. B.E. Jones and R.C. Spooncer, "An Optical Fibre Pressure Sensor Using a Holographic Shutter Modulator with Two-Wavelength Intensity Referencing," in Conference Proceedings OFS ' 84 - Second International Conference on Optical Fiber Sensors, R.T. Kersten and R. Kist, eds., Proc. SPIE 514, pp. 223-226, 1984.

14. G. Adamovsky, "Time Domain Referencing in Intensity Modulation Fiber Optic Sensing Systems," in Optical Testing and Metrology, C.P. Grover, ed., Proc. SPIE 661, pp. 145-151, 1986.

15. W.B. Spillman, Jr. and J.R. Lord, "Self-Referencing Multiplexing Technique for Fiber-Optic Intensity Sensors," J. Lightwave Technol, vol. LT-5. pp. 865-869, July 1987.

16. G. Adamovsky and N.D. Piltch, "Fiber-Optic Thermometer Using Temperature Dependent Absorption, Broadband Detection, and Time Domain Referencing. "Appl. Opt., vol. 25, pp. 4439-4443, Dec. 1, 1986.

17. G. Adamovsky, T.N. Sherer, and D.J. Maitland, "Intensity-Based Fibre-Optic Sensing System Using Contrast Modulation of Subcarrier Interference Pattern," Electron. Letters, vol. 25, pp. 325-326, Mar. 2, 1989.

18. W. Nakwaski and A.M. Kontkiewicz, "Temperature Distribution in a Light-Emitting Diode During a Pulse Operation," Electron. Letters, vol. 20, pp. 984-986, Nov. 8, 1984.

19. W.H. Glenn, W.W. Morey, and R.M. Elkow, "Design, Fabrication, and Testing of an Optical Temperature Sensor for High Temperature Control Applications," NASA CR-174698, 1984 .

20. R.A. Fields, M. Birnbaum, and C.L. Fincher, "Highly Efficient Nd:YVO 4 DiodeLaser End-Pumped Laser," Appl. Phys. Lett., vol. 51, pp. 1885-1886, Dec. 7, 1987. 
21. H.P. Jenssen, R.F. Begley, R. Webb, and R.C. Morris, "Spectroscopic Properties and Laser Performance of $\mathrm{Nd}^{3+}$ in Lanthanum Beryllate," J. Appl. Phys., vol. 47, pp. 1496-1500, Apr. 1976.

22. J.R. O'Connor, "Unusual Crystal-field Energy Levels and Efficient Laser Properties of $\mathrm{YVO}_{4}: \mathrm{Nd}, "$ Appl. Phys. Lett. vol. 9, pp. 407-409, Dec. 1, 1966.

TABLE I

\begin{tabular}{|r|c|c|c|c|c|c|}
\hline \multirow{2}{*}{${ }^{\circ} \mathrm{C}}$, & \multicolumn{3}{|c|}{$\lambda_{\mathrm{C}}=820 \mathrm{~nm}, \Delta \lambda=30 \mathrm{~nm}$} & \multicolumn{3}{c|}{$\lambda_{\mathrm{C}}=820 \mathrm{~nm}, \Delta \lambda=100 \mathrm{~nm}$} \\
\cline { 2 - 7 } & $\mathrm{Ta}=-40{ }^{\circ} \mathrm{C}$ & $\mathrm{Ta}=25{ }^{\circ} \mathrm{C}$ & $\mathrm{Ta}=75{ }^{\circ} \mathrm{C}$ & $\mathrm{Ta}=-40^{\circ} \mathrm{C}$ & $\mathrm{Ta}=25{ }^{\circ} \mathrm{C}$ & $\mathrm{Ta}=75{ }^{\circ} \mathrm{C}$ \\
\hline 0 & 0.222 & 0.369 & 0.543 & 0.501 & 0.506 & 0.501 \\
100 & .184 & .306 & .481 & .453 & .457 & .454 \\
200 & .160 & .267 & .438 & .419 & .422 & .419 \\
300 & .144 & .241 & .407 & .392 & .394 & .391 \\
\hline
\end{tabular}

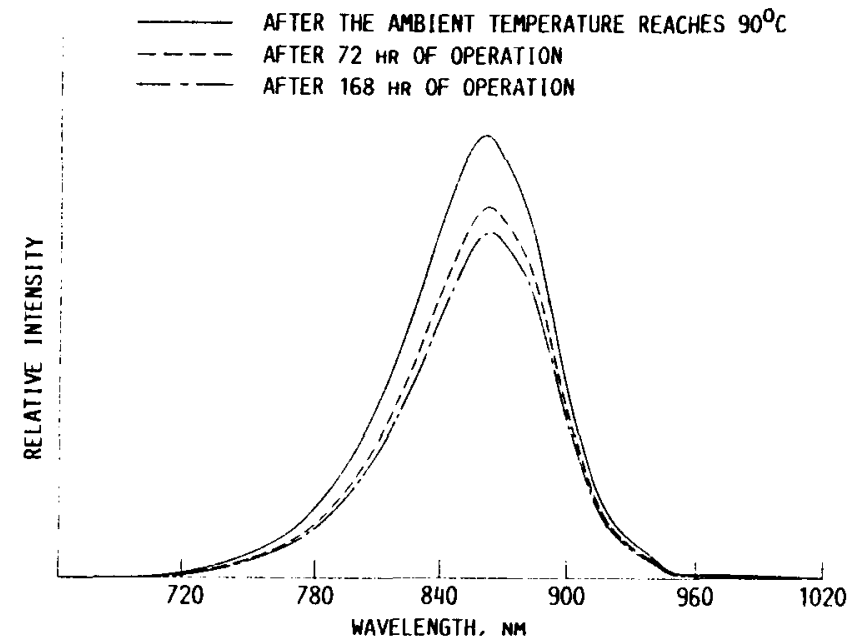

FIGURE 1. - RELATIVE SPECTRAL INTENSITY DISTRIBUTIONS OF LED OPERATED AT 100 MA CONTINOUS CURRENT AND AMBIENT TEMPERATURE $T_{d}=90^{\circ} \mathrm{C}$.

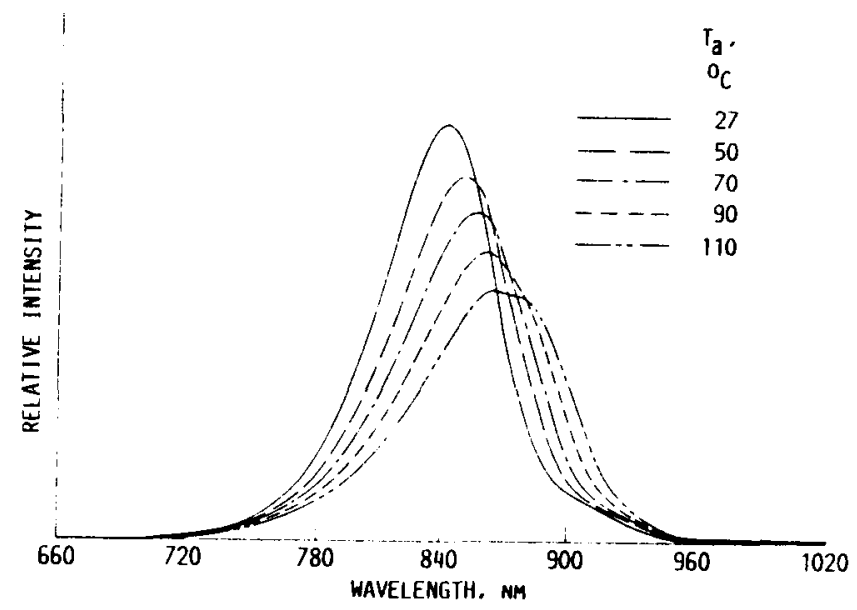

FIGURE 2. - RELATIVE SPECTRAL IMTENSITY DISTRIBUTIONS OF LED AT DIFFERENT AMBIENT TEMPERATURE $\mathrm{J}_{\mathrm{a}}$. 


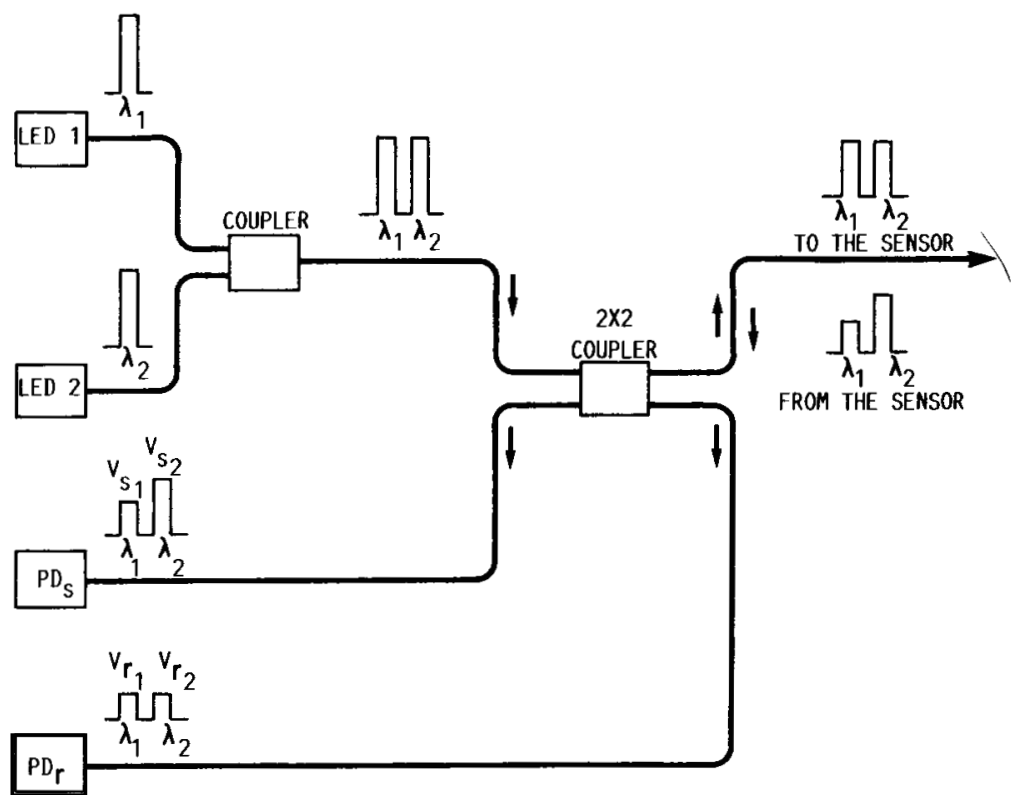

FIGURE 3. SCHEMATIC OF SENSING SYSTEM WITH DUAL WAVELENGTH REFERENCING AND POWFR LEVEL COMPENSATION.

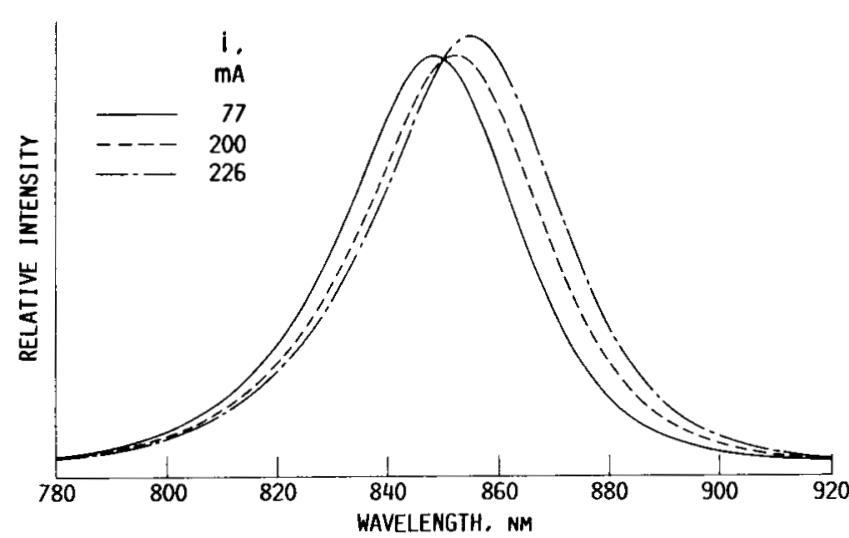

FIGURE 4. - RELATIVE SPECTRAL INTENSITY DISTRIBUTIONS OF LED OPERATED AT ROOM TEMPERATURE AND DIFFERENT CONTINOUS CURRENTS $\mathrm{i}$.

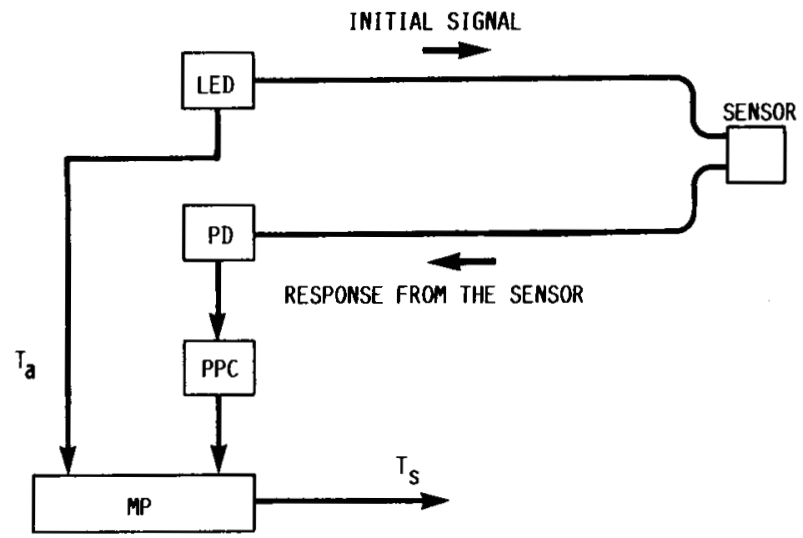

FIGURE 5. - SCHEMATIC OF INDIRECT PASSIVE COMPENSATION TECHNIQUE. 


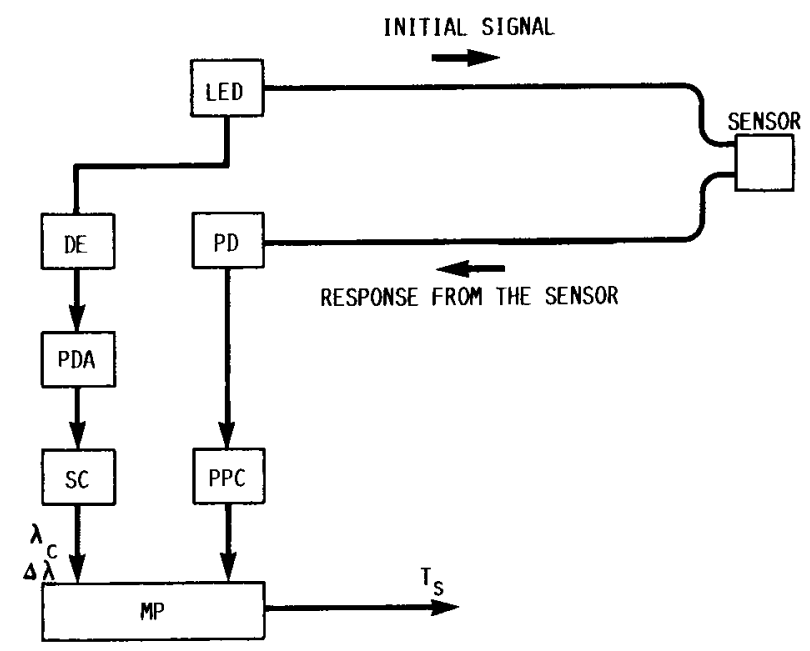

FIGURE 6. - SCHEMATIC OF DIRECT PASSIVE COMPENSATION TECHNIQUE.

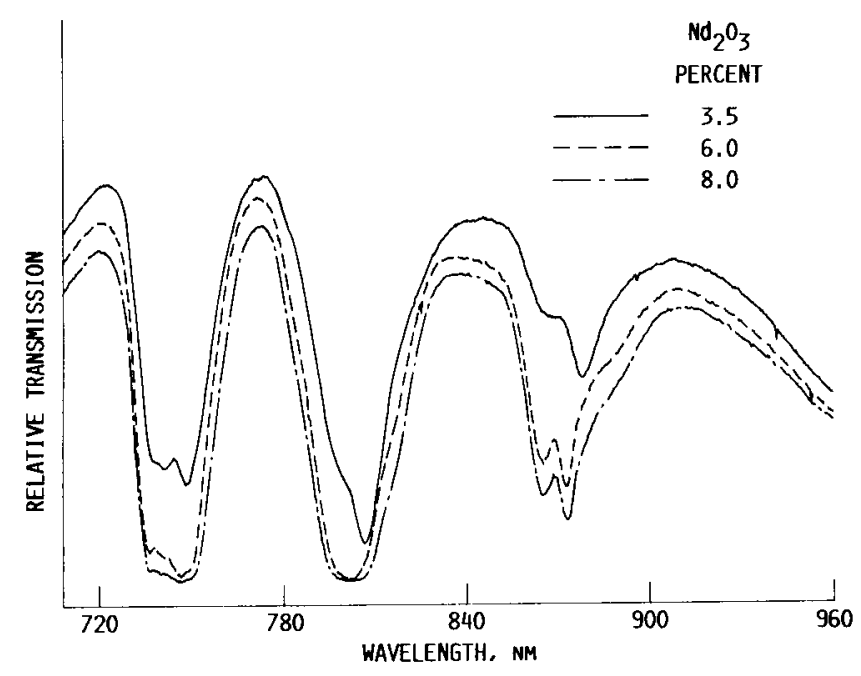

FIGURE 7. - RELATIVE TRANSMISSION SPECTRA OF GLASS SAMPLES WITH DIFFERENT CONCENTRATIONS OF $\mathrm{Nd}_{2} \mathrm{O}_{3}$ AT ROOM TEMPERATURE. 


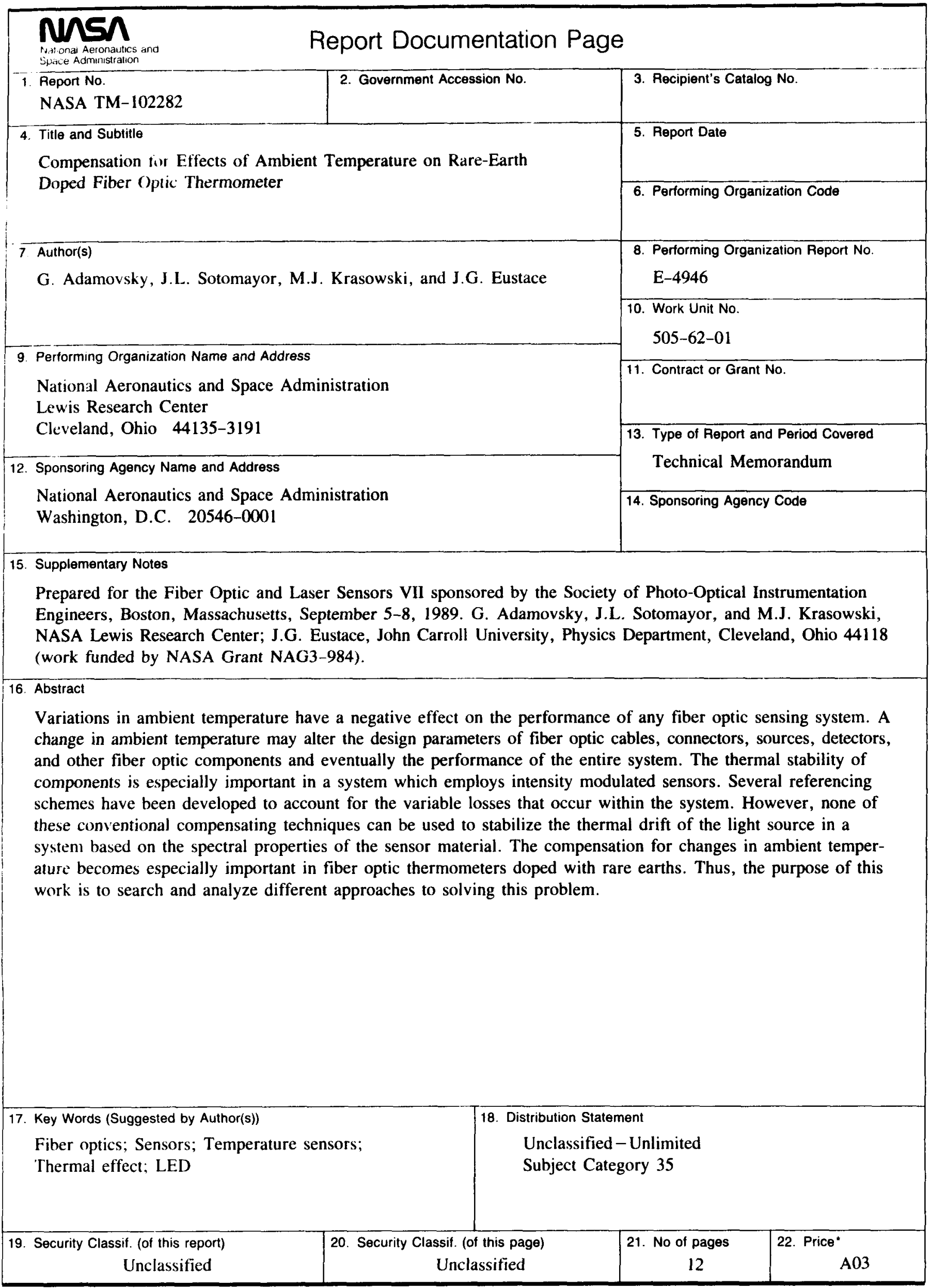

